\title{
Giant planet formation
}

\section{A first classification of isothermal protoplanetary equilibria}

\author{
B. Pečnik ${ }^{1,2}$ and G. Wuchterl ${ }^{2}$ \\ 1 Max-Planck-Institut für extraterrestrishe Physik, Postfach 1312, 85748 Garching, Germany \\ 2 Astrophysikalisches Institut und Universitäts- Sternwarte, Schillergächen 2-3, 07745 Jena, Germany \\ e-mail: bonnie@astro.uni-jena.de
}

Received 7 May 2003 / Accepted 16 December 2004

\begin{abstract}
We present a model for the equilibrium of solid planetary cores embedded in a gaseous nebula. From this model we are able to extract an idealized roadmap of all hydrostatic states of the isothermal protoplanets. The complete classification of the isothermal protoplanetary equilibria should improve the understanding of the general problem of giant planet formation, within the framework of the nucleated instability hypothesis. We approximate the protoplanet as a spherically symmetric, isothermal, self-gravitating classical ideal gas envelope in equilibrium, around a rigid body of given mass and density, with the gaseous envelope required to fill the Hill-sphere. Starting only with a core of given mass and an envelope gas density at the core surface, the equilibria are calculated without prescribing the total protoplanetary mass or nebula density. In this way, a variety of hydrostatic core-envelope equilibria has been obtained. Two types of envelope equilibria can be distinguished: uniform equilibrium, were the density of the envelope gas drops approximately an order of magnitude as the radial distance increases to the outer boundary, and compact equilibrium, having a small but very dense gas layer wrapped around the core and very low, exponentially decreasing gas density further out. The effect of the envelope mass on the planetary gravitational potential further discriminates the models into the self-gravitating and the non-self gravitating ones. The static critical core masses of the protoplanets for the typical orbits of 1, 5.2, and $30 \mathrm{AU}$, around a parent star of 1 solar mass $\left(M_{\odot}\right)$ are found to be $0.1524,0.0948$, and 0.0335 Earth masses $\left(M_{\oplus}\right)$, respectively, for standard nebula conditions (Kusaka et al. 1970). These values are much lower than currently admitted ones primarily because our model is isothermal and the envelope is in thermal equilibrium with the nebula. Our solutions show a wide range of possible envelopes. For a given core, multiple solutions (at least two) are found to fit into the same nebula. Some of those solutions posses equal envelope mass. This variety is a consequence of the envelope's self-gravity. We extend the concept of the static critical core mass to the local and global critical core mass. Above the global critical mass, only compact solutions exist. We conclude that the "global static critical core mass" marks the meeting point of all four qualitatively different envelope regions.
\end{abstract}

Key words. planets and satellites: general - solar system: general

\section{Introduction}

With the discovery of the extra-solar gas giants, the general problem of planet formation has considerably grown in complexity over the last decade. However, a global theoretical overview of the properties of giant planets, irrespective of the parent protoplanetary disc or the total mass of the giant planet, is still missing.

In the nucleated instability hypothesis, envelopes of giant planets are thought to be formed as a consequence of accretion of solid bodies forming their cores. To determine the envelope mass corresponding to a given core, static protoplanetary models have been constructed (e.g. Perri \& Cameron 1974; Mizuno 1980; Stevenson 1982).

If the envelope is modelled including detailed energy transfer and if the outer part of the envelope is radiative, and for standard assumptions about nebula conditions, it has been found that there is an upper limit for the masses of static envelopes and therefore for the total mass of a proto giant planet. This upper limit in core mass - the critical mass - was found to be insensitive to nebula conditions, but to depend weakly on dust opacities (Mizuno 1980) and on the rate at which the core (solid body) is accreted (Stevenson 1982).

Even the largest static critical masses are typically more than a factor of ten smaller than Jupiter's mass (Mizuno 1980; Stevenson 1982; Wuchterl 1991b; Ikoma et al. 2001). The nondependence of the critical mass on nebula conditions disappears when the outermost parts of the protoplanetary envelopes become convective, which happens for nebula properties which are well within of proposed solar nebula conditions (Wuchterl 1993). Envelope masses of such protoplanets range between 6 and 48 Earth masses $\left(M_{\oplus}\right)$ but hydrostatic models alone are unable to reproduce a Jupiter-mass protoplanet. Therefore 
dynamical and/or quasi-hydrostatical effects should play an important role in the formation of gas giants.

There is a number of incompletely studied processes (e.g. the formation, evolution, and stability of the protoplanetary disks, the dust growth, the planetesimal formation, etc.) that are relevant for the general problem of planet formation. Their complexity makes a piecewise approach necessary in studies of planet formation. An alternative approach is to study the final outcome, i.e. the possible and probable end-states of the process. In that context, we present an idealized roadmap of all hydrostatic states, in order to provide insight when analyzing the complex behavior of hydrodynamic and quasihydrostatic models with detailed microphysics. In addition, this work aims to clarify the concept of the critical core mass necessary to permanently attract gas of the protoplanetary nebula to a terrestrial-planet-like heavy element core.

\section{Model}

\subsection{Motivation}

Within nucleated instability theory, the formation of giant planets includes many possible scenarios for protoplanetary cores and their respective envelopes. These range from small planetoids embedded in dilute protoplanetary nebulae to presentday-Jovian-like cores of several $M_{\oplus}$ squeezed by some Mbars of metallic $\mathrm{H}_{2}$-He mixtures (Guillot 1999). To date, many investigations have been made into the evolution of protoplanets, both hydrostatically (e.g. Bodenheimer et al. 2000; Ikoma 2001; see Wuchterl et al. 2000, for review) and hydrodynamically (e.g. Wuchterl 1991a,b, 1993). In these studies, "the evolution" of particular planets is followed, but not much is known about the evolution of all possible protoplanets. Therefore, it is somewhat difficult to bring the detailed solutions of previous investigations within a global perspective.

We follow the thermodynamical approach that was used by Stahl et al. (1995) to investigate the coreless equilibria of constant-mass isothermal gas spheres, and the nature of the Jeans instability. We also expand on the work of Sasaki 1989, who studied isothermal protoplanets in the minimum mass solar nebula (MMSN). In our model the total mass of the protoplanet and the density of nebula cloud, in which the protoplanet is embedded, are not prescribed. In leaving these as output variables, and starting only with the (heavy-element) core mass and the density of the envelope gas at the core's surface, we aim for a complete classification of all hydrostatic equilibria. This classification should contribute to clarifying whether multiple planetary equilibria exist for given nebula conditions and how protoplanetary models relate to gas giants, both inside and outside the solar system.

\subsection{Model assumptions}

We approximate the protoplanet as a spherically symmetric, isothermal, self-gravitating classical ideal gas envelope in equilibrium around a core of given mass. This gaseous envelope
Table 1. Symbols.

\begin{tabular}{ll}
\hline \hline Symbol $^{\dagger}$ & Meaning \\
\hline$a[\mathrm{AU}]$ & orbital distance \\
$G=6.67259 \times 10^{-11}$ & gravitational constant \\
$\mu=2.3 \times 10^{-3}$ & mean molecular weight \\
$M_{\text {core }}$ & predefined core mass \\
$M_{\text {env }}$ & envelope mass \\
$M_{\text {tot }}$ & total mass \\
$M(r)$ & total mass interior to radius $r$ \\
$M_{\odot}=1.989 \times 10^{30}$ & solar mass \\
$M_{\oplus}=5.976 \times 10^{24}$ & Earth mass \\
$r_{\text {core }}$ & core radius \\
$r_{\text {Hill }}$ & Hill sphere radius \\
$\mathfrak{R}=8.31441$ & molar gas constant \\
$\varrho_{\text {core }}=5500$ & predefined core density \\
$\varrho_{\text {csg }}$ & envelope gas density at core surface \\
$\varrho_{\text {env }}$ & envelope gas density \\
$T(a)$ & nebula gas temperature \\
\hline
\end{tabular}

$\dagger$ SI units used unless otherwise specified.

Table 2. Manifolds.

\begin{tabular}{lrrr}
\hline \hline Orb. param. $(a, T)$ & $(1,225)$ & $(5.2,123)$ & $(30,51.1)$ \\
\hline$M_{\text {core,crit }}^{\text {MMSN }} /\left[M_{\oplus}\right]$ & 0.1524 & 0.0948 & 0.0335 \\
$M_{\text {env }}^{\text {max }} /\left[M_{\oplus}\right]$ & 21 & 96 & 380 \\
\hline
\end{tabular}

The critical core mass increases for smaller orbital distances because of (in order of importance): the higher gas temperature (cf. Sects. 3.8.1 and 3.10), the smaller Hill sphere (cf. Sect. 3.8.2), and the higher densities of the reference nebulae (taken from the minimum mass solar nebula models of Kusaka 1970; and Hayashi 1985).

is that required to fill the gravitational sphere of influence, approximated by the Hill-sphere:

$r_{\text {Hill }}=a \sqrt[3]{M_{\text {planet }} / 3 M_{\star}}$

where $a$ is the orbital distance from a parent star. With mean molecular weight of $\mu=2.3 \times 10^{-3} \mathrm{~kg} \mathrm{~mol}^{-1}$, protoplanetary envelopes, as well as the nebula, are roughly approximated by a hydrogen-helium mixture. The protoplanet's heavy-elementcore is represented by a rigid sphere of uniform density of $\varrho_{\text {core }}=5500 \mathrm{~kg} \mathrm{~m}^{-3}$.

The nebula temperature profile is taken according to Kusaka et al. (1970), and Hayashi et al. (1985), cf. Table 2. The nebula density structure is not a priori determined, but, for critical core mass determination, nebula densities agree with those from Kusaka et al. (1970) for $a=1$ and $30 \mathrm{AU}$, and from Hayashi (1985) for $a=5.2 \mathrm{AU}$, cf. Table 2. It has been shown that the critical core mass values have only a weak dependence on the nebula density (cf. Sect. 3.9), therefore the choice of the nebula density is not critical. 


\subsection{Model equations}

The envelope is set in isothermal hydrostatic equilibrium, with spherical symmetry, and as such is described by:

$\frac{\mathrm{d} M(r)}{\mathrm{d} r}=4 \pi r^{2} \varrho(r)$

the equation of hydrostatic equilibrium:

$\frac{\mathrm{d} P(r)}{\mathrm{d} r}=-\frac{G M(r)}{r^{2}} \varrho(r)$,

and the equation of state for an ideal gas:

$P(r)=\frac{\mathfrak{R} T}{\mu} \varrho(r)$.

$M(r)$ is defined as the total mass (core plus envelope) contained within the radius $r$ :

$M(r)=M_{\text {core }}+\int_{r_{\text {core }}}^{r} 4 \pi r^{\prime 2} \varrho\left(r^{\prime}\right) \mathrm{d} r^{\prime}$,

where $r$ is the radial distance measured from the core center and $\varrho$ is the envelope gas density at radial distance $r$.

\subsection{Boundary conditions}

The total mass of the protoplanet is defined as:

$M_{\mathrm{tot}}=M_{\mathrm{core}}+M_{\mathrm{env}}=M\left(r_{\mathrm{out}}\right)$

with

$M\left(r_{\text {core }}\right)=M_{\text {core }}$.

The inner and outer radial boundaries are:

$r_{\text {in }}=r_{\text {core }}=\sqrt[3]{\frac{M_{\text {core }}}{\frac{4}{3} \pi \varrho_{\text {core }}}} \quad$ and $\quad r_{\text {out }}=r_{\text {Hill }}$.

An additional boundary condition at the core surface is:

$\varrho_{\text {env }}\left(r_{\text {core }}\right)=\varrho_{\text {csg }}$

This model, together with the specified assumptions and boundary conditions, is sufficient to completely determine a single model-protoplanet. The total mass and nebula density at $r_{\text {Hill }}$ (gas density at protoplanet's outer boundary) are results of the calculation.

\subsection{Solution procedure}

The total protoplanetary mass is obtained by integrating outward from $r_{\text {core }}$ to $r_{\text {Hill }}\left(M_{\text {tot }}\right)$, starting with $r_{\text {Hill }}^{0}=r_{\text {Hill }}\left(M_{\text {core }}\right)$ and iterating $r_{\text {Hill }}\left(M_{\text {core }}+M_{\text {env }}\right)$.

Integration is performed from the core surface to the Hill radius, using the Maple 6 software (e.g. Garvan 2001), with the Fehlberg fourth-fifth order Runge-Kutta method.

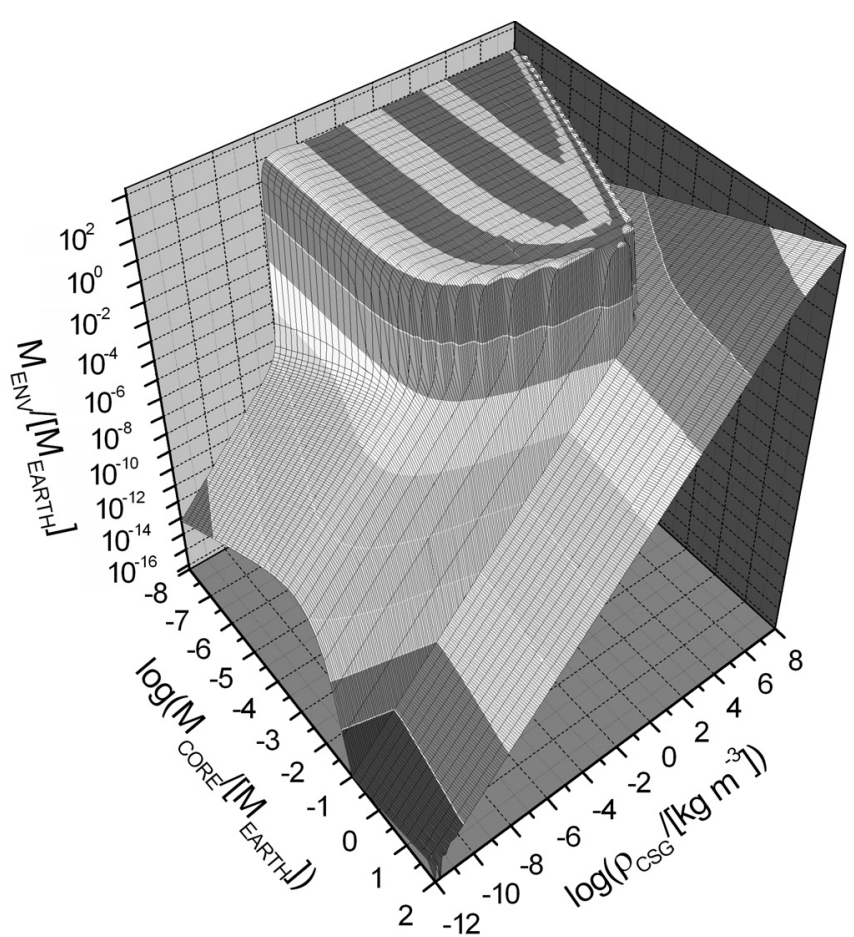

Fig. 1. Envelope mass solution manifold. Environmental parameters for this manifold are set to $a=5.2 \mathrm{AU}$, and $T=123 \mathrm{~K}$. Each point on the surface gives the mass of the protoplanet's envelope for a given $M_{\text {core }}$ and gas density at the core surface, $\varrho_{\text {csg }}$. Models with different initial parameters generally connect to different nebulae. Several different regions are easily discernible: I - flat slope with gradient of 1 , for the region $[-1,2]$ in $\log M_{\text {core }}$ and $[-12,6]$ in $\log \varrho_{\text {csg }}$; II - flat slope with gradient of 0.5 , roughly encompassing [4-6, 8] in $\log \varrho_{\text {csg }}$, and all $\log M_{\text {core }}$; III - "base of the island", $[-8,-1]$ in $\log M_{\text {core }}$ and $[-12,-6]$ in $\log \varrho_{\text {csg }}$; IV - "island", $[-8,-1]$ in $\log M_{\text {core }}$ and $[-6,4-8]$ in $\log \varrho_{\text {csg }}$ (cf. Fig. 2).

\section{Results}

\subsection{Solution manifold}

In order to cover as many hydrostatic solutions as possible, the system of Eqs. (2)-(4) is solved for a wide range of parameters $M_{\text {core }}$ and $\varrho_{\text {csg }}$. The set of all solutions for this range constitutes the solution manifold. Figure 1 shows the solution manifold for a protoplanet whose orbital distance corresponds to the position of proto-Jupiter according to the Kyoto-model of solar system formation (Hayashi et al. 1985). The manifolds with orbital parameters $(a, T)$ of proto-Neptune and proto-Earth have similar morphologies. It should be reiterated that the solution set contains all qualitatively different protoplanetary models at a particular orbital distance; not just for a particular nebula, but for any nebula, from a dense gravitationally-just-stable cloud to a near-vacuum space.

\subsection{Manifold regions}

Several distinct regions exist in the parameter space of the solution manifold (Fig. 2), and they can be examined from two complementary perspectives. One way is to use the gas density at the core surface, $\varrho_{\text {csg }}$, as an independent variable (e.g. 


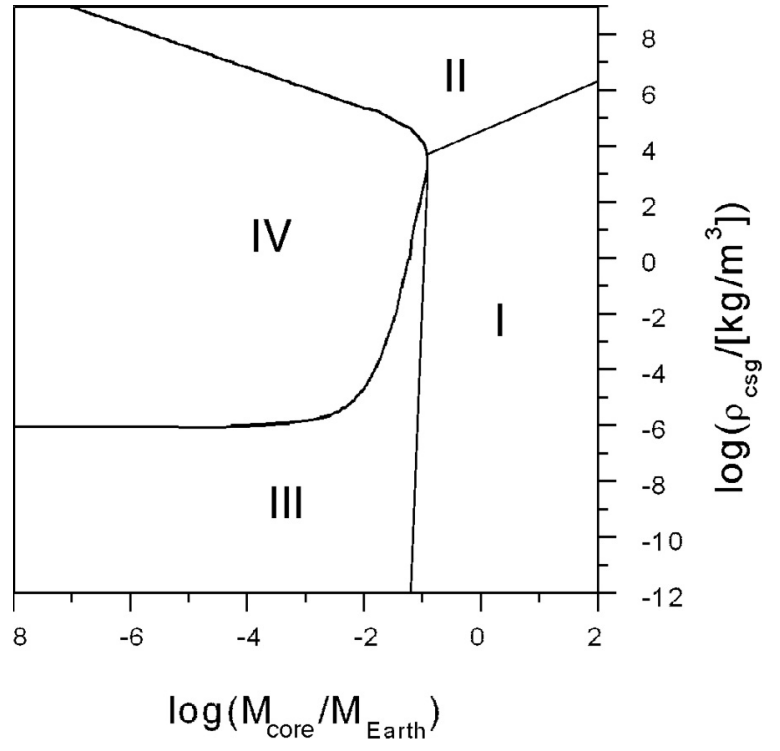

Fig. 2. Manifold regions: I - compact non-self-gravitating envelopes, II - compact self-gravitating envelopes, III - uniform non-selfgravitating envelopes, IV - uniform self-gravitating envelopes. The border of the region IV somewhat depends on the choice of the surrounding nebula (cf. Fig. 12); we use here a value from the Hayashi (1985) minimum mass solar nebula model.

Fig. 3), and the other is to use the nebula gas density, $\varrho_{\text {out }}$ (e.g. Fig. 4). While $\varrho_{\text {out }}$ is more physically intuitive, $\varrho_{\text {csg }}$ maps out region IV of Fig. 2 more clearly, and is more efficient in terms of representing the entire manifold.

Figure 2 divides the solution manifold into four distinct regions, depending on whether the solution is compact or uniform and self-gravitating or not. Figures 3 and 4 point to the existence of the four possible regimes for a planet;

1. "mature telluric planet" (region I): envelope mass is a linear function of $\varrho_{\text {out }}$, and $\varrho_{\mathrm{csg}}$;

2. "mature giant planet" (region II): envelope mass weakly drops with $\varrho_{\text {out }}\left(M_{\text {env }} \propto \varrho_{\text {out }}^{-0.005}\right)$.

$M_{\text {env }} \propto \varrho_{\text {csg }}^{0.5}$ is weaker than for the "nebula" regime. "Nebula" densities $\left(\varrho_{\text {out }}\right)$ are so low that they may well be considered a vacuum;

3. "nebula"(region III): envelope mass is a linear function of $\varrho_{\text {out }}$ and $\varrho_{\mathrm{csg}}$;

4. "protoplanet" (region IV): envelope mass is a non-trivial function of $\varrho_{\text {out }}$ or $\varrho_{\text {csg }}$.

\subsection{Self-gravity effect}

The key effect, which is responsible for the manifold morphology as observed in Fig. 1, can be described as self-gravity of the protoplanet's envelope. Keeping in mind the hydrostatics of the model, and the fact that the surrounding nebula is not prescribed, one can see that self-gravity reduces the envelope mass for a given core surface pressure, i.e. the envelope mass would be larger if there were no self-gravitating effect (Fig. 3).

The envelope's radial gas density profile is shaped through the interplay of inward gravitational force and outward gas

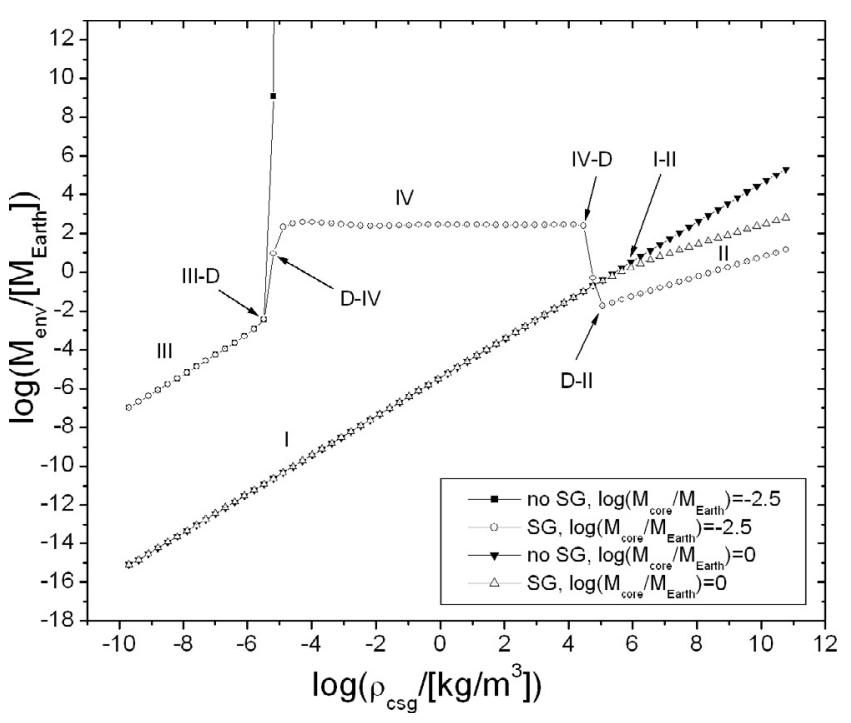

Fig. 3. Demonstration of the self-gravitating effect for sub- and supercritical cores: comparison of cuts through two manifolds, with- $(M=$ $M(r)$ in Eq. (3)) and without- $\left(M=M_{\text {core }}\right)$ the envelope's gravitating effect, each for two core masses. Cuts are for $a=30 \mathrm{AU}$ and $T=51.1 \mathrm{~K}$. Circles and squares represent the envelope mass of the subcritical core, calculated for $M=M(r)$ and $M=M_{\text {core }}$ in Eq. (3), respectively. White and black triangles have the same meaning but for the supercritical core. Labels without arrows correspond to manifold regions from Fig. 2, while labels with arrows mark interfaces between regions. $D$ corresponds to the "divergent wall" which surrounds region IV (cf. Fig. 1). Self-gravitating envelopes with $M=M(r)$ in Eq. (3) have a larger envelope mass than the corresponding envelopes with $M=M_{\text {core }}$ in Eq. (3) (cf. Fig. 6).

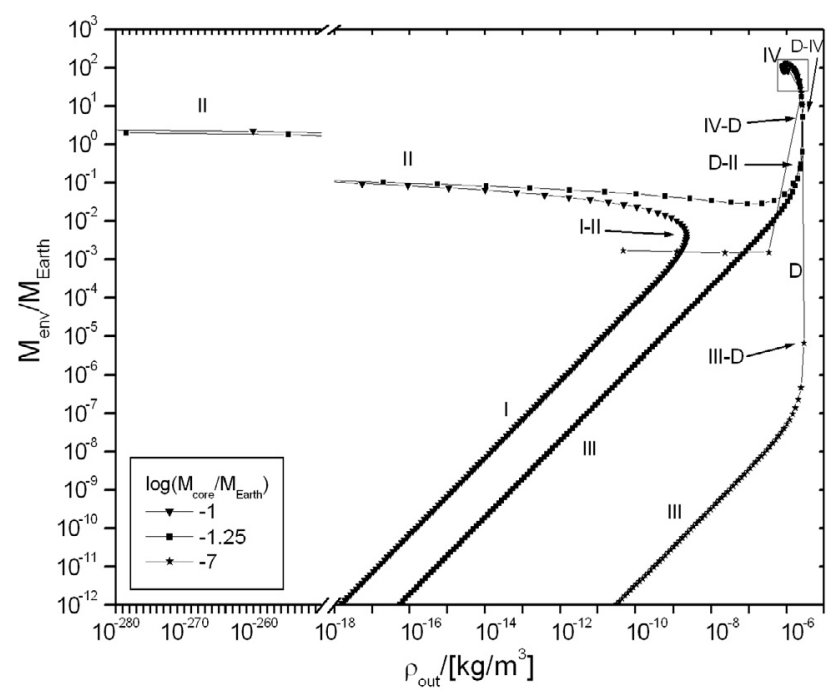

Fig. 4. Envelope mass as a function of the nebula density $\varrho_{\text {out }}$. Labels are the same as in Fig. 3. Lines connect states with increasing $\varrho_{\text {csg }}$. Note the strong dependence of $\varrho_{\text {out }}$ on the envelope mass, and a nontrivial behavior of the $M_{\text {env }}\left(\varrho_{\text {out }}\right)$ for region IV (enlargement in Fig. 5).

pressure. If the envelope mass is small compared to the core mass, the gravitational force can be approximated as arising from the core's gravitational potential only. Once the envelope mass is comparable to (or greater than) the core mass, they both contribute to the gravitational potential, making its 


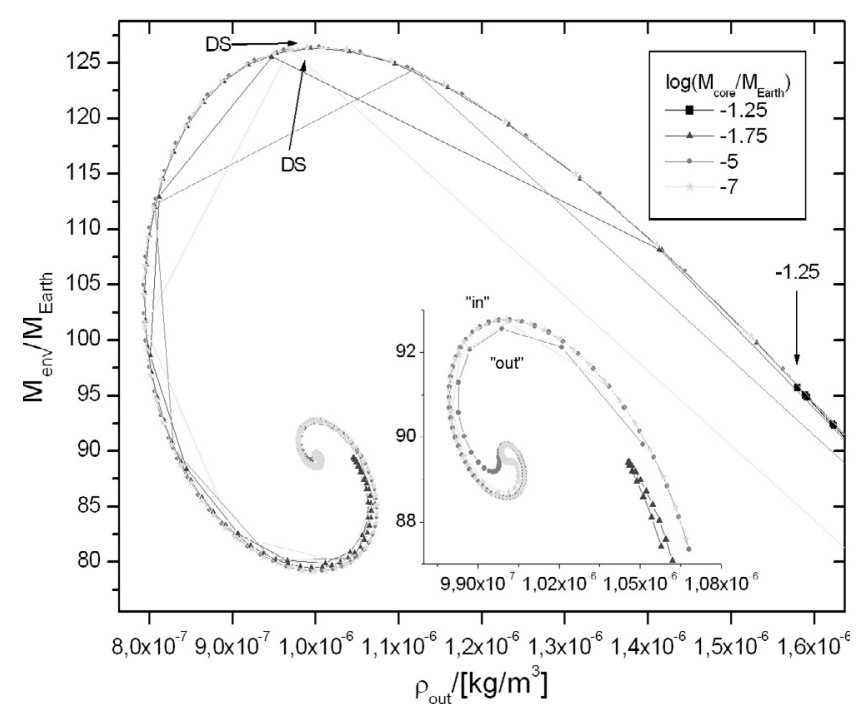

Fig. 5. Enlargement of the boxed region of Fig. 4, isothermal curl regularized with the finite-density core; "-1.25": black squares represent protoplanets with first subcritical $M_{\text {core }}$ line on the mesh of Fig. 1 and the arrow points at the black square with the highest $M_{\text {env }}, D S$ : two protoplanetary states with the largest envelope mass in the manifold, but with typically very different $\varrho_{\mathrm{csg}}$ (cf. Sect. 3.9.2); in and out curves are the consequence of the core. The smaller the core, the closer the in and out curves are. The figure corresponds to a $V-U$ plane for the protoplanets (see Sect. 3.5 for further discussion).

gradient steeper and, in effect, reducing the envelope mass. As a consequence, the self-gravitating envelope connects to a nebula different from the one which is in balance with the envelope in the absence of the self-gravitating effect. Further discussion of the role of self-gravity can be found in Sect. 3.5.

\subsection{Two types of envelope equilibria}

The solution manifold (Fig. 2) contains two basic types of envelope equilibria (Fig. 6):

1. uniform, or quasi-homogenous envelope: the density of the envelope gas drops weakly with increasing radial distance, keeping the mass distribution more or less uniform throughout the envelope; $\partial M_{\text {env }} / \partial r_{\text {out }}>0$;

2. condensed, or quasi-compact envelope: typically small, but very dense gas layer is wrapped around the core, at larger radii the gas density is very low; $\partial M_{\text {env }} / \partial r_{\text {out }} \approx 0$.

This is reminiscent of a similar equilibrium, found by Stahl et al. (1995), for constant mass coreless "Van der Waals" gas spheres.

If an envelope's mass is much smaller than the core mass, the radial profile of the gas density is simply an exponential function, well approximated by:

$P(r)=P_{0} \exp \left(-\frac{\mu}{\mathfrak{R} T} G M(r)\left(\frac{1}{r_{\text {core }}}-\frac{1}{r}\right)\right)$.

If $\left(M(r)-M_{\text {core }}\right) \ll M_{\text {core }}$, then Eq. (10) reduces to the barometric formula.

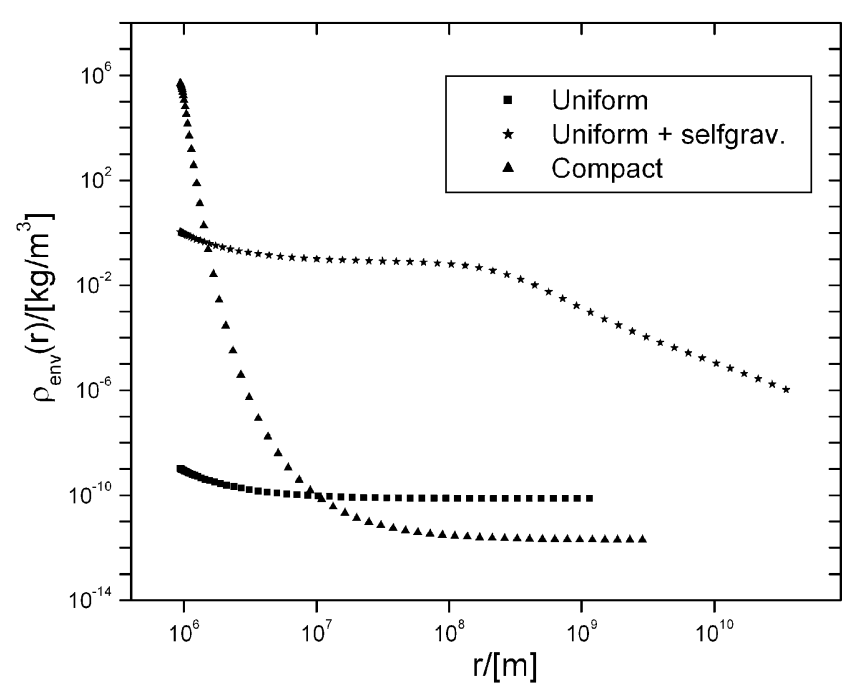

Fig. 6. Uniform, compact and self-gravitating profiles. The uniform self- gravitating profile resembles the non-self-gravitating one until the envelope mass becomes comparable to the core mass. Then the density profile changes to $\varrho_{\text {env }}(r) \propto r^{-2}$.

\subsection{Differences: isothermal coreless gas spheres vs. isothermal protoplanets}

The curl in Figs. 4 and 5 is reminiscent of a similar feature found for the isothermal coreless ideal-gas spheres (e.g. Schwarzschild 1957, Sect. 13) represented in the $U-V$ plane. It follows from the definition of $U$ and $V$ that:

$U=\frac{r}{M(r)} \frac{\mathrm{d} M(r)}{\mathrm{d} r}=\frac{4 \pi r^{3} \varrho}{M(r)}=3 \frac{\varrho}{M(r) /\left(\frac{4}{3} \pi r^{3}\right)}$
$V=-\frac{r}{P} \frac{\mathrm{d} P}{\mathrm{~d} r}=\frac{\varrho}{P} \frac{G M(r)}{r}=\frac{3}{2} \frac{G M(r) / r}{\frac{3}{2} P / \varrho}$

and from the fact that the mean density of the total object for our model is always the same, as implicitly defined through Eq. (1).

Unlike a singular isothermal sphere with an infinite pressure at the center, our protoplanetary model has a solid core of uniform (and finite) density at its center. This will result in the departure from the potential of the coreless isothermal sphere: instead of having a $\varrho(r) \propto r^{-2}$ structure, the envelope gas close to the core surface will obey the form of the barometric law (cf. Eq. (10)).

If the mean envelope density at lower stratifications is comparable to core density, an "effective" core will shorten the characteristic length-scale of the potential, making the exponential profile of the barometric-law-like profile even steeper. For the appropriate effective core, the outer stratifications will exactly match the outer stratifications of the solution which has the gas density at the core surface much smaller than the core density (cf. Fig. 14). These profiles will connect to the same nebula density, but will have a slightly different envelope mass, because of the difference in the profile of the inner stratification. Therefore, the curl of Fig. 5 will have two branches: "in" (the solution with a non-self-gravitating inner stratification) and "out" (the solution with an effective core). 
The smaller the core mass, the sooner will the profile connect to the " $r^{-2}$ " structure; i.e. the smaller the difference in the envelope mass between the pairs of solutions, the closer the "in" and "out" branches in Fig. 5 will be.

The fall-off of the gas density with increasing radius in the self-gravitating part of the envelope can be approximated by $\varrho_{\text {env }}(r) \sim r^{-2}$ (cf. Fig. 6, self-gravitating profile), as expected in the theory of stellar structure for a self-gravitating isothermal sphere of ideal gas (e.g. Shu 1992, Sect. 18). Small deviations from $r^{-2}$ are due to the finite amount of mass needed for the envelope to become self-gravitating, which produces a slight imbalance between the self-gravity and the amount of mass $M(r)$. No similar effect is observed for coreless, isothermal gas spheres (cf. Stahl et al. 1995).

Depending on the fraction of the self-gravitating part of the envelope and of the core mass, this wavelike deviation can extend to the outer boundary, or can be attenuated deep within the envelope.

\subsection{Estimating the applicability of the ideal gas}

We made two major assumptions while constructing our model - that the gas is ideal, and that the heat is instantaneously radiated away, i.e. the gas is isothermal. In Sect. 3.7 we examine the isothermal assumption, and we deal with the ideal gas in this section.

In order to keep the protoplanet in an equilibrium with the surrounding nebula, we have set the envelope gas temperature equal to the nebula temperature for the appropriate orbital distance. Therefore, we compare different equations of state at the envelope temperature. In addition to an ideal gas, we take the Saumon-Chabrier-van Horn (1995) EOS, the CarnahanStarling (1969) EOS, as well as a completely degenerate electron gas.

Figure 7 shows that for gas densities up to about $40 \mathrm{~kg} \mathrm{~m}^{-3}$ ideal gas, Saumon-Chabrier-van Horn, and Carnahan-Starling EOS agree to better than one percent. For higher densities the Saumon-Chabrier EOS shows additional non-ideal effects, while the Carnahan-Starling EOS exhibits a similar behavior for densities larger than $200 \mathrm{~kg} \mathrm{~m}^{-3}$. We can also see that the electron degeneracy does not contribute to the pressure at least till the point where the Saumon-Chabrier EOS departs from ideal-gas behavior.

However, in general we see that the ideal gas is an excellent approximation for our model for the better part of the envelope gas density range. Certainly, there are also models where densities are high enough for significant non-ideal effects, but typically for the protoplanets in our model those high density envelope regions are restricted to areas close to the core, while the rest of the envelope will be well approximated by an ideal gas. We can see in Fig. 8 that if we use e.g. the CarnahanStarling EOS, the numerical details will be changed, but the qualitative picture will remain the same. This is also true for the Saumon-Chabrier EOS, which is work in preparation by C. Broeg. The ideal isothermal gas will not be a good approximation for the compact envelopes which are typically associated with giant planets in the late stages of their evolution. Using

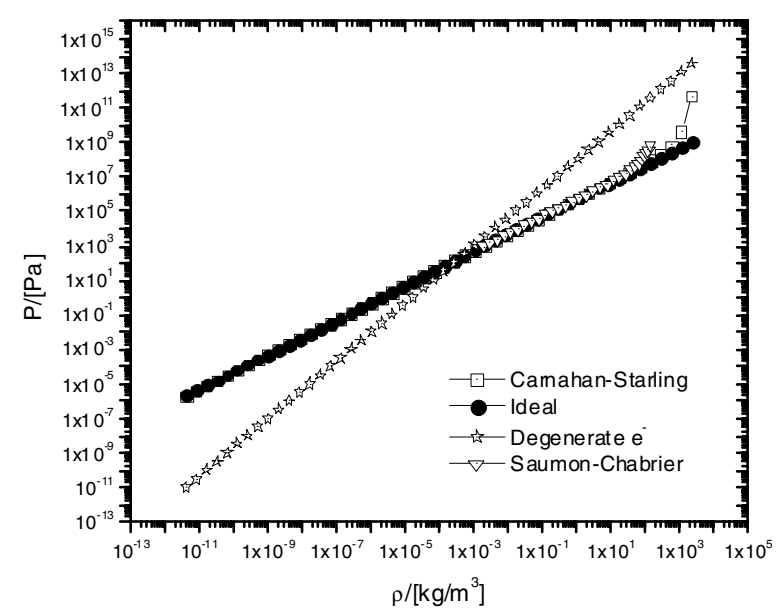

Fig. 7. Pressure as a function of density, for $T=123 \mathrm{~K}$. Black circles represent the ideal gas, squares are for the Carnahan-Starling EOS, and triangles are for the Saumon-Chabrier EOS. This figure also shows that a completely degenerate electron gas (stars) is not a good assumption for this $(\varrho, T)$ parameter range.

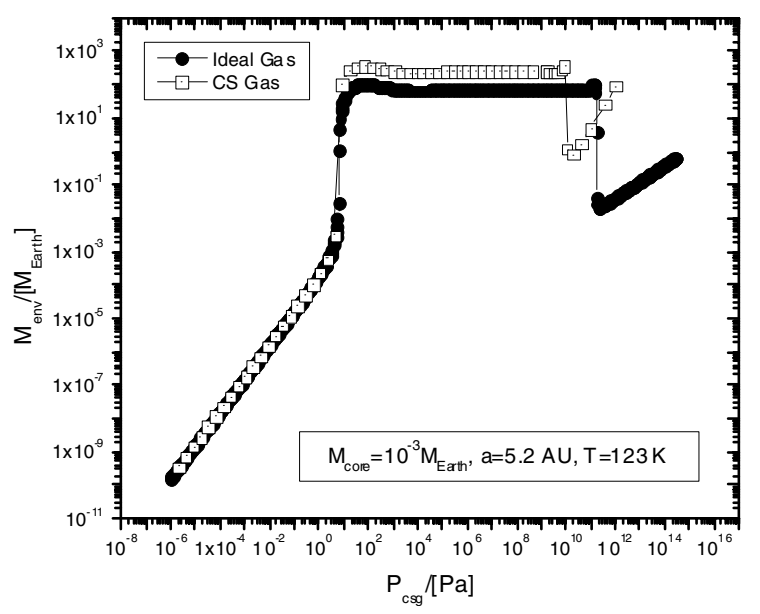

Fig. 8. Cut through the envelope mass manifold, for a $10^{-3} M_{\otimes}$ core, $a=5.2 \mathrm{AU}$, and $T=123 \mathrm{~K}$. Black circles represent the ideal gas, and squares are for the Carnahan-Starling EOS.

our model, we can show that a protoplanet will have a compact envelope under certain conditions. What we cannot do with this model is obtain a quantitatively correct picture of such a compact envelope.

Additionally, Fig. 14 shows why the choice of EOS is not critical for the qualitative picture: Although the non-ideal effects might change the density stratifications of the compact inner parts, each solution which is not self-gravitating in its inner (barometric-law like) part, will have a counterpart solution with an effective core. Properties of the effective core will be dictated by the EOS, but its effect on the scale-height will remain the same. 


\subsection{Estimating the applicability of the isothermal assumption}

In the previous section we showed that an ideal gas is a good approximation for most of the parameter range we use. The validity of the isothermal assumption is examined below.

By analogy with the pressure scale-height, a temperature scale-height of a radiative stratification can be defined as:

$H_{T}=\frac{H_{P}}{\nabla_{\mathrm{rad}}}=-\frac{\partial r}{\partial \ln T}$

where

$H_{P}=-\frac{\partial r}{\partial \ln P}=\frac{P}{\varrho} \frac{r^{2}}{G M(r)}$

for an ideal gas and hydrostatic equilibrium, and

$\nabla_{\mathrm{rad}}=-\left.\frac{\partial \ln T}{\partial \ln P}\right|_{\mathrm{rad}}=\frac{3 \kappa L P}{4 \pi a c G M(r) T^{4}}$,

where $a$ is the radiation constant, $\kappa$ is the gas opacity taken to be $0.1 \mathrm{~m}^{2} \mathrm{~kg}^{-1}, c$ is the speed of light, and $L$ is the core luminosity due to the planetesimal accretion rate of $10^{-6} M_{\oplus} \mathrm{yr}^{-1}$.

The temperature scale-height corresponds to the lengthscale of a radiative giant-protoplanet over which the envelope temperature drops by a factor of $1 / \mathrm{e}$. The specific temperature scale-height $H_{T}(r) / r_{\text {Hill }}$ evaluates the ratio of the thermal length-scale to the radial extent of the entire protoplanetary envelope, at a position $r$. Evaluated at $r=r_{\mathrm{Hill}}, H_{T}\left(r_{\mathrm{Hill}}\right)$ is the global estimate of the thermal scale-height of the protoplanet. Figure 9 shows that the isothermal assumption is valid for large portions of the manifold regions III and IV (cf. Fig. 2), where $H_{T}\left(r_{\text {Hill }}\right) / r_{\text {Hill }}$ has values much larger than unity. These envelopes have a specific thermal scale-height above unity for at least the outer $90 \%$ of the envelope. Therefore, even though the small innermost envelope region is probably nonisothermal, the protoplanet should be well represented by the isothermal gas.

Close to the giant-protoplanet's critical core mass (e.g. $\log M_{\text {core }}=-1.25$ in Fig. 4$), H_{T} / r_{\text {Hill }}$ is expected to be of order unity and the isothermal assumption breaks down. Compact solutions (regions I and II from Fig. 2, and high $\varrho_{\text {csg }}$ solutions in Fig. 9) have very large $H_{T} / r_{\text {Hill }}$, indicating that nearlyvacuum space around the compact envelope is nearly isothermal. Detachment from a protoplanetary nebula could represent either hydrodynamically active protoplanets, or the collapsed gas giants with cleared protoplanetary nebula (i.e. mature giant planets). In both cases objects are expected to be deep in the non-isothermal regime. The radial profiles of the compact objects will change if a detailed energy transport is included, but they will nevertheless remain compact. A comparison of Jupiter's radius with that of our model planet (of equivalent mass and $T_{\text {env }}$ of $5000 \mathrm{~K}$, estimated to be representative of Jupiter's average temperature from Guillot 1999) shows that, with $r_{\text {compact }}=6.63 \times 10^{7} \mathrm{~m}$, our model falls short by less than $10 \%$ of reproducing the radius of the real gas giant.

In the context of Jupiter's potentially rapid formation (order of $10^{6}$ years), it could be argued that the core accretion rate should be even higher. However, $H_{T} / r_{\text {Hill }}$ is proportional to the

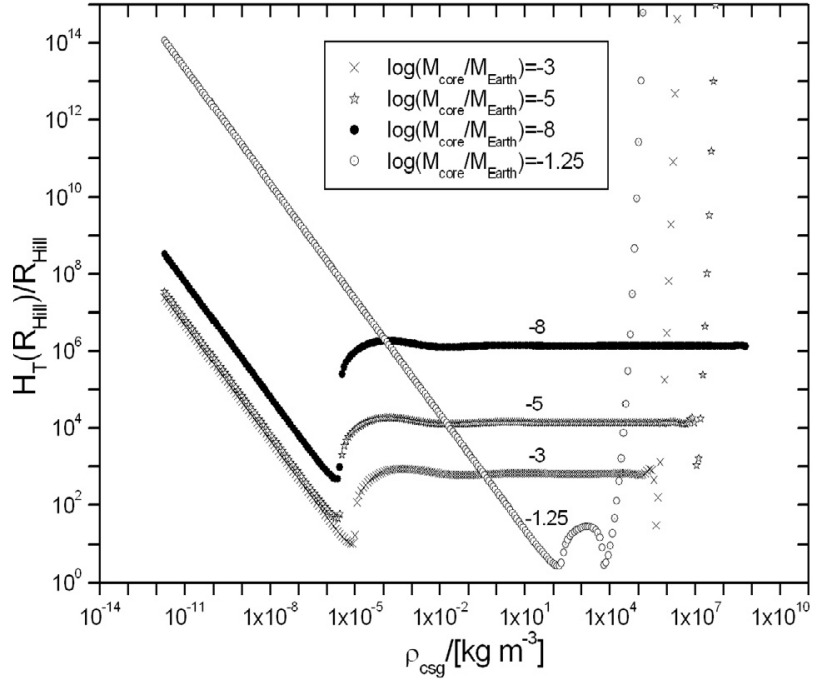

Fig. 9. Specific temperature scale-height as a function of the density at the core surface, for different subcritical core masses. Protoplanetary models with cores of -8 (black circles), -5 (stars), and -3 (crosses) in logarithmic $M_{\oplus}$ units have $H_{T}\left(r_{\text {Hill }}\right) / r_{\text {Hill }}$ much larger than unity. This justifies the isothermal assumption for the manifold regions III and IV.

inverse of $\dot{M}_{\text {core }}$, and even if it is set to $10^{-5} M_{\oplus} \mathrm{yr}^{-1}$, the validity of the isothermal assumption is still appropriate for the regions III and IV of Fig. 2. Indeed, such high core accretion rates are applicable for cores comparable to $M_{\oplus}$ (i.e. cores at late stages of a giant protoplanet's evolution), and are surely an overestimation for the younger cores (e.g. for the cores of $10^{-3} M_{\oplus}$ ), making the case for the isothermal regime even more solid. However, because of the simplicity of our model, the results are only qualitative, while quantitatively correct values would only be accessible through a more elaborate model.

$H_{T} / r_{\text {Hill }}$ shows that close to the critical core mass there are non-isothermal effects.

But the basic isothermal picture is valid for most of (the quasi-homogenous part of) the manifold. It even appears that the possible transition from homogenous to compact state can be initiated within the isothermal regime.

\subsection{Manifolds and environment}

Manifold solutions are dependent on four environmental parameters: the gas temperature $T$ of the protoplanet (and of the surrounding nebula), the orbital distance $a$ from the parent star, the mean molecular weight $\mu$, and the mass of the parent star $M_{\star}$. These parameters influence the balance of the two forces that determine the radial density structure - the outward force arising from the gas pressure, and the inward force of gravity; $T$ and $\mu$ are connected with pressure through Eq. (4), while $a$ and $M_{\star}$ determine the Hill-sphere, i.e. the volume of the envelope mass.

Because of the simplicity of the model, the impact of $T$ and $\mu$ on the solutions will be discussed together, as will the influence of $a$ and $M_{\star}$. In reality, these parameters will have very different impact. 


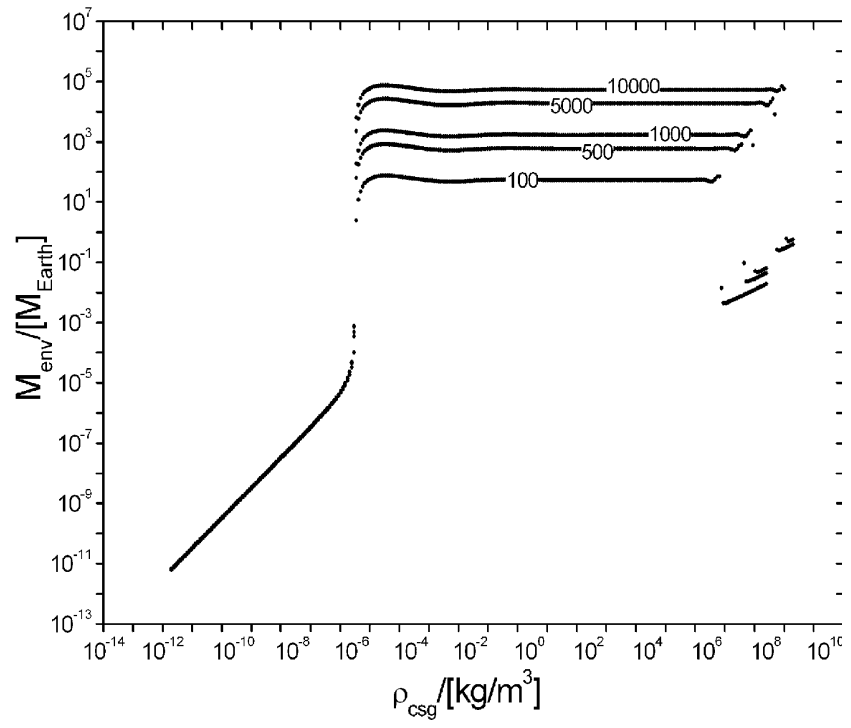

Fig. 10. Envelope mass solutions as a function of gas density at the core surface, for gas temperatures of $100,500,1000,5000$, and $10000 \mathrm{~K}$. A change of $T$ has no influence on the envelope mass of the non-self-gravitating regions, while the same change of $T$ will produce a significant effect for protoplanets in self-gravitating regions.

Unless otherwise specified, the reference parameters throughout the current section are: $\log \left(M_{\text {core }} / M_{\oplus}\right)=-5, a=$ $5.2 \mathrm{AU}, T_{\text {env }}=123 \mathrm{~K}$, and $\mu=2.310^{-3} \mathrm{~kg} \mathrm{~mol}^{-1}$.

\subsubsection{Temperature and mean molecular weight}

Although this model is isothermal, the choice of gas temperature influences the solution manifold quantitatively. From Eq. (4) it is clear that pressure relates linearly to temperature. Since the pressure force counterbalances the gravitational force, protoplanets with hotter envelopes require more gravity (and thus more mass) for a hydrostatic solution. The value of the critical core mass is a good example of the quantitative influence of the temperature. For example, the critical core mass for a $123 \mathrm{~K}$ protoplanet in Jupiter's orbit is $0.0948 M_{\oplus}$, while the critical core mass value for a $5000 \mathrm{~K}$ case is $24.5 M_{\oplus}$.

Figure 10 shows that, for subcritical cores and low gas densities at the core surface (region III in Fig. 2), the gas temperature has virtually no impact on the envelope mass. Since the envelope mass is small compared to the core mass, the envelope parameters (e.g. $T_{\text {env }}$ ) have no influence on the hydrostatic force balance via gravity feedback. On the contrary, for envelopes in which self-gravity shapes the radial structure (regions IV and II in Fig. 2), the envelope mass is significantly affected by different $T_{\text {env }}$.

The scaling law which relates manifolds of various temperatures is discussed in Sect. 3.10.

As previously mentioned, this simple model does not incorporate an energy transport equation, nor does it take into account the gas and dust opacities. Therefore, a change in $\mu$ cannot be distinguished from the corresponding change in $T$, and will not be further discussed.

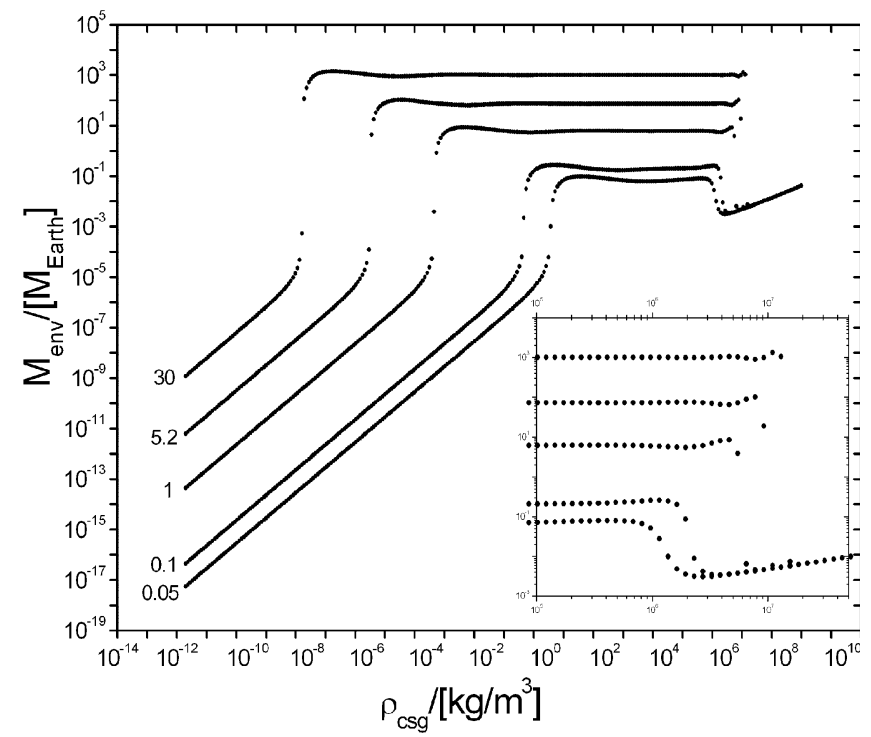

Fig. 11. Envelope mass solutions as a function of gas density at the core surface, for orbital distances of $0.05,0.1,1,5.2$, and $30 \mathrm{AU}$. Enlargement: the transition from uniform to compact envelope solutions is more abrupt for protoplanets at large orbital radii. This is a consequence of the larger Hill-sphere of outer protoplanets.

\subsubsection{Orbital distance and star class}

Orbital distance, together with the masses of the protoplanet and the parent star determine the protoplanet's gravitational sphere of influence, the so called Hill-sphere. Since the available volume for the protoplanet's envelope scales with the cube of the orbital distance (see Eq. (1)), the strength of the envelope's self-gravitating effect depends critically on the distance from the core to the parent star (see Fig. 11). Therefore, for the inner protoplanets to have (at least partly) self-gravitating envelopes, the gas density at the core surface must be larger than for the corresponding outer protoplanets.

For solutions with compact envelopes (right side of Fig. 11 and enlargement) the orbital distance has no impact on the envelope mass, since the radii of the compact inner part are typically several orders of magnitude smaller than their respective Hill-spheres.

The transition from a uniform self-gravitating to a compact envelope is characterized by a considerable drop in the gas density for the outer envelope stratifications. In addition, protoplanets close to the parent star have relatively small Hill-radii and most of the envelope mass can be found in the proximity of the core. Therefore, the transition from uniform to compact envelope for protoplanets close to the parent star is less abrupt than for more distant protoplanets, as can be seen in the enlargement of Fig. 11.

Varying the mass of the parent star is equivalent to changing the orbital distance of the protoplanet, provided that the gas temperature stays the same. It follows from Eq. (1) that $\delta a^{-3}=$ $\delta M_{\star}$, e.g. changing the orbital distance of the protoplanet from $5.2 \mathrm{AU}$ to $1 \mathrm{AU}$ is equivalent to changing the mass of the parent star from $M_{\star}=0.21 M_{\odot}$ to $M_{\star}=30 M_{\odot}$. It remains to be seen whether this equivalence will hold for a more complex model, 


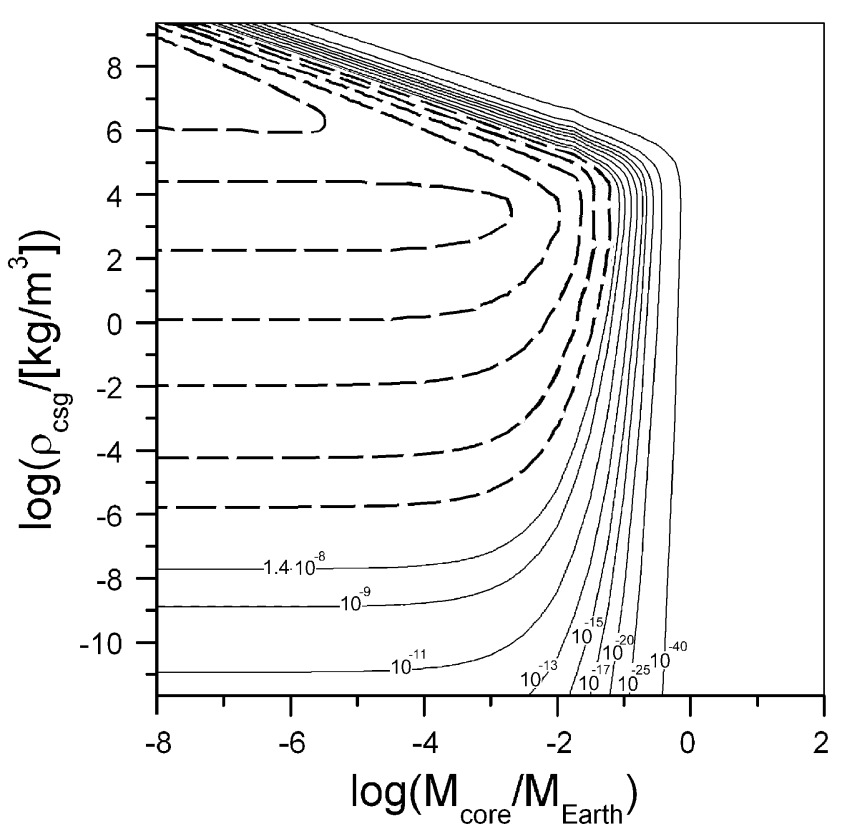

Fig. 12. Solution branches - isobars for $\varrho_{\text {env }}\left(r_{\text {Hill }}\right)=\varrho_{\text {out }}-$ for $(a=$ 5.2 AU and $T=123 \mathrm{~K}$ ) manifold: the standard solar nebula solution branch is represented by the innermost solid line; an enhanced nebula with $\varrho_{\text {out }}=10^{-6} \mathrm{~kg} \mathrm{~m}^{-3}$ (dashed lines) has multiple solution branches; each solution branch has its own maximum core mass, and hence local critical mass.

because the nebula properties will likely change in accordance with the known mass-luminosity relation as $M_{\star}$ is varied.

\subsection{Static critical core mass}

There are several definitions of the critical core mass currently in use. The critical core mass concept has been introduced by various investigators (e.g. Perri \& Cameron 1974; Mizuno et al. 1978; Mizuno 1980; Bodenheimer \& Pollack 1986; Wuchterl 1991a). As a starting point, we choose here a definition suggested by Wuchterl (1991a), for "static critical core mass": No more static core-envelope models with increasing core mass exist at the critical mass.

This definition is valid along a (time) sequence of protoplanetary models with increasing $M_{\text {core }}$. It is only along such a sequence, in the context of the static models, that a time evolution with growing cores can proceed. Essentially, the static critical core mass is the largest core mass for a static protoplanet that can be embedded in a given nebula, characterized by a nebula gas density, a temperature, and a distance from a parent star.

For the ( $a=5.2 \mathrm{AU}$ and $T=123 \mathrm{~K})$ manifold this means that, among the solutions with $\varrho_{\text {env }}\left(r_{\text {Hill }}\right)=1.4 \times 10^{-8} \mathrm{~kg} \mathrm{~m}^{-3}$ (defined for the minimum mass solar nebula, e.g. Hayashi et al. 1985), the solution with the largest core mass determines the static critical mass (Fig. 12, the innermost solid line). This gives a static critical core mass of $M_{\text {core,crit }}^{\mathrm{MMNN}}=0.0948 M_{\oplus}$.

Figure 12 shows that the value for the critical core mass exhibits a generally weak dependence on the density of the surrounding nebula, so the choice of $\varrho_{\text {out }}^{\mathrm{MMN}}$ from different nebula models is not critical. For the very dense nebulae (around
$10^{-6} \mathrm{~kg} \mathrm{~m}^{-3}$ ) and depending on the choice of the solution branch (cf. Sect. 3.9.1), the values for the local critical core masses can span several orders of magnitude even for the same nebula.

The critical core masses for different manifolds are presented in Table 2, and are found to depend on the parameters that affect the hydrostatic balance (cf. Sect. 3.8).

By comparing Figs. 1 and 2 it follows that the natural choice for the global static critical core mass, one which is valid for the whole manifold, should be the core of the protoplanet which is at the interface of all four manifold regions (cf. Fig. 2). The model at the interface has a minimum in the envelope mass, for a manifold cut along the constant $\varrho_{\text {csg }}$ value. The interface is also an inflection point for a manifold cut at a constant $M_{\text {core }}$. The conditions for the global static critical core mass thus are:

$$
\begin{array}{ll}
\frac{\partial M_{\text {env }}}{\partial M_{\text {core }}}=0 & \frac{\partial^{2} M_{\text {env }}}{\partial M_{\text {core }}^{2}}>0 \\
\frac{\partial M_{\text {env }}}{\partial \varrho_{\text {csg }}}=0 & \frac{\partial^{2} M_{\text {env }}}{\partial \varrho_{\text {csg }}^{2}}=0 .
\end{array}
$$

Since the numerical values for the global critical core masses are very close to the values of the critical core masses from the definition suggested by Wuchterl (1991a), we do not present the global numerical values separately.

The values obtained for critical core masses in this model agree well with those of Sasaki (1989), who used a similar set of assumptions. However, such isothermal values are significantly smaller than today's commonly accepted critical mass values, obtained with the inclusion of detailed energy transfer, which are typically between 7 and $15 M_{\oplus}$. The reasons for this are two-fold. Firstly, we use the equation of state for an ideal gas. Secondly, the temperature of the isothermal gas is taken from nebula models, hence the nebula temperature is the temperature of the entire protoplanet. This is certainly a lower limit for a realistic temperature of the interior of the protoplanet. Larger critical core mass values are obtained if the gas temperature is in the range of the temperatures for the interior of gas giants modelled with detailed energy transfer (cf. Sect. 3.8.1). Clearly, the correct determination of the critical core mass requires temperature structure, but the emphasis in this work was not on quantitative details, but rather on global qualitative features.

\subsubsection{Local critical core mass}

From Figs. 12 and 13 one can see that, for each subcritical core immersed in a nebula, there are at least two permitted solutions. However, if one considers only the time-sequence of hydrostatic models with a growing core, it is clear that solutions with higher density at the core surface cannot be reached.

The situation is more complicated if the protoplanet is embedded in a denser protoplanetary nebula. Our model clearly predicts multiple solutions for certain sets of parameters (Fig. 13). Instead of one solution branch for a given nebula (with two solutions for each core, as for a minimum-mass solar nebula), several solution branches are possible, again each with two solutions for a specific core (Fig. 12, dashed solution 


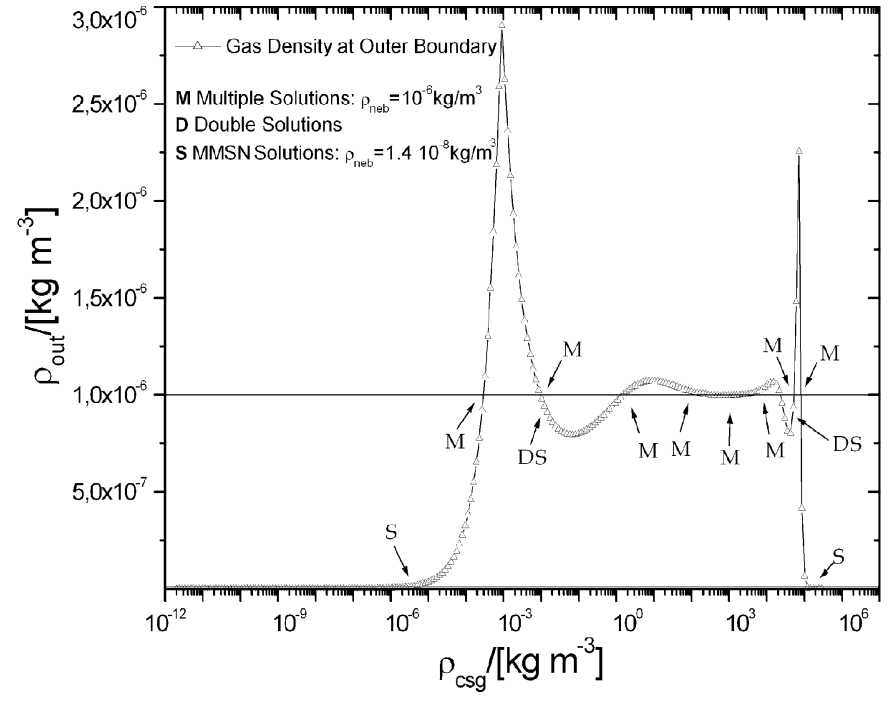

Fig. 13. For nebula density enhanced relative to a minimum-mass solar nebula, even more than two hydrostatic equilibria could exist; $M$ : protoplanetary solutions with $\log M_{\text {core }} /\left[M_{\text {Earth }}\right]=-2$ that fit into $\varrho_{\text {out }}=10^{-6} \mathrm{~kg} \mathrm{~m}^{-3}$ nebula; $D S$ : double solutions, a special case of multiple solutions, cf. Figs. 5 and 15; $S$ : protoplanetary solutions with the same core, whose envelope fits into the minimum-mass solar nebula.

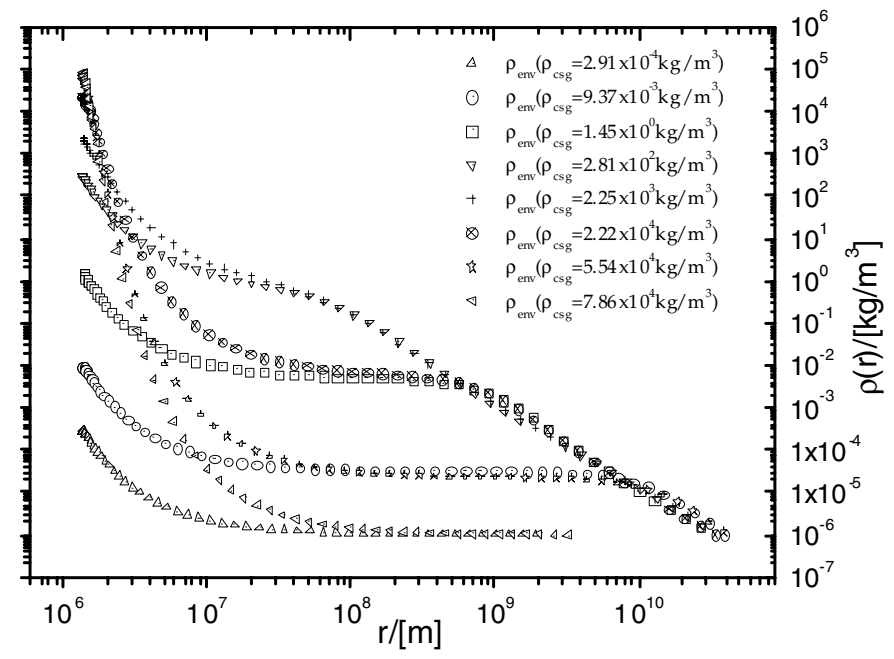

Fig. 14. Density profiles for the solutions which fit into the same $\left(10^{-6} \mathrm{~kg} \mathrm{~m}^{-3}\right)$ nebula. These solutions are labelled with $M$ in Fig. 13.

branches for $\varrho_{\text {out }}=10^{-6} \mathrm{~kg} \mathrm{~m}^{-3}$ ). Multiple solution-branches are enabled by envelope self-gravity (cf. Fig. 14) and are due to tidal restrictions imposed by the parent star via $r_{\text {Hill }}$ (cf. Fig. 3, region IV).

Each solution branch has one critical core mass, beyond which there is no static solution, for a sequence of hydrostatic models with increasing core mass. For the minimum mass solar nebula this means one critical core mass, according to the definition suggested by Wuchterl (1991a). For some denser nebulae, however, the existence of several branches implies several local critical core masses, where solutions beyond the critical core mass of the branch are unavailable locally. After reaching the local critical core mass, the planet could, in principle, continue evolving by 'jumping' to another branch. One of us has

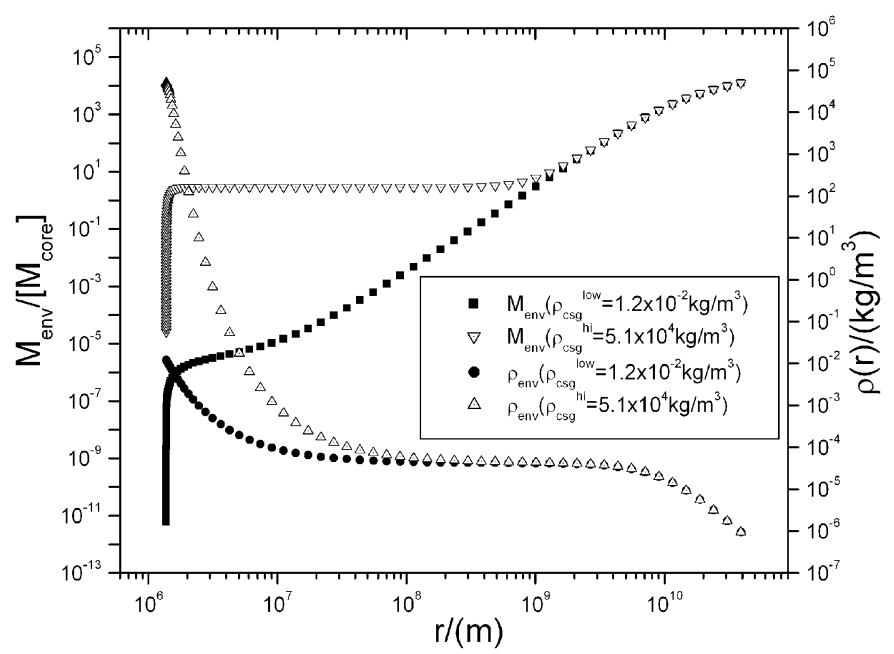

Fig. 15. Mass and density radial structure of the special case of multiple solutions, where two protoplanets have the same core, almost the same envelope mass, connect to the same nebula, but have different radial structure. These solutions are labelled $D S$ in Fig. 13.

observed similar behavior for certain sets of initial parameters in hydrodynamical models. The local critical core mass satisfies the above definition but not Eq. (16) for the global critical core mass.

\subsubsection{Double maxima}

A special case of multiple solutions can be seen in Figs. 5, 13, and 15 as double peaks in the envelope mass. For every (subcritical) core, two special solutions, which fit into the same nebula cloud (i.e. have the same $\left.\varrho\left(r_{\text {Hill }}\right)\right)$ and have almost exactly the same envelope mass (equal to one part in $10^{4}$, or better), are found to exist. Usually these two solutions have a very similar stratification in the outer parts of the protoplanet's envelope, but deep inside the protoplanet their radial structure is quite different (cf. Sect. 3.5).

Supercritical cores do not posses such a feature, because the density profile always effectively goes to zero long before the Hill radius is reached. Therefore there is no significant contribution to the envelope mass in the outer stratifications, and the envelope mass increases monotonically with the gas density at the core surface (cf. Fig. 3).

Envelopes with lower gas density at the core surface, $\varrho_{\text {csg }}^{\text {low }}$, (Fig. 15) have a maximum possible mass (for the corresponding manifold) because the envelope gas density at the core surface is low enough to ensure uniformity for the major part of the radial structure. Consequently, the envelope density does not substantially decrease from the core-surface value. At the same time, $\varrho_{\mathrm{csg}}$ is high enough to allow significant mass contributions from the outer parts of the envelope, where the volume (and therefore the mass, for a given density) per unit radius, is the largest. Values for such maximum envelope masses are tabulated in Table 2.

Envelopes with higher $\varrho_{\mathrm{csg}}^{\text {high }}$ build up the self-gravitating effect (SG effect starts as soon as $M_{\text {env }} \approx M_{\text {core }}$ ) very close to their core. Because of the very strong self-gravitating effect 
( $M_{\mathrm{env}} \approx 3 M_{\text {core }}$ for the innermost regions), the radial density fall-off close to the core (Fig. 15) is strong.

A new, effective core is formed from the dense envelopegas-layer wrapped tightly around the core. In this case, the envelope density distribution resembles one with the core (and the radius) of this effective core. In stratifications where the envelope mass becomes comparable to the effective core, another self-gravitating effect changes the radial envelope density distribution to $\varrho_{\text {env }} \propto r^{-2}$.

For a particular choice of $\varrho_{\text {csg }}^{\text {high }}$, the envelope density profile in the outer stratifications matches that of $\varrho_{\text {csg }}^{\text {low }}$, thus making the mass of both envelopes almost equal.

\subsection{Temperature-mass invariance}

It has been noted that, if mass and distance are measured in a system of appropriate units (i.e. mass in units of core mass, and distance in units of core radii), solution manifolds with different temperatures are almost identical, except for a shifting on a core-mass-axis, according to the relation:

$\frac{T_{1}}{T_{2}}=\left(\frac{M_{1}}{M_{2}}\right)^{2 / 3}$

that can be derived for homologous envelopes satisfying $\varrho_{1}\left(r_{1} / r_{\text {core }, 1}\right)=\varrho_{2}\left(r_{2} / r_{\text {core }, 2}\right)$, for any pair of $r_{1}$ and $r_{2}$ such that $r_{1} / r_{\text {core }, 1}=r_{2} / r_{\text {core, } 2}$. In other words, the radial profile of a certain protoplanet with core mass $M_{1}$ and temperature $T_{1}$ will be the same as the radial profile of another protoplanet with core mass $M_{2}$ and temperature $T_{2}$, if Eq. (17) is obeyed, and if the mass is measured in units of core mass and the length in units of core radius.

This is true for all manifold regions, sub- and supercritical, self-gravitating or not. Note that in Fig. 10 the non-self-gravitating region was not affected by a change in envelope temperature, but relation (17) does hold even for nonself-gravitating envelopes, since it connects envelopes with different temperatures and core masses. Figure 10 was plotted for different temperatures, but constant core mass.

\section{Discussion and conclusions}

In an effort to obtain a global overview of hydrostatic protoplanetary equilibria, we have chosen a simple physical model so as to be able to clearly understand the interaction of competing processes.

Our use of relatively simple physics has several consequences; because the ideal gas equation of state is used, gas particles are "soft", and can be compressed as much as is needed, in effect overestimating the importance of gravity relative to gas pressure, when large envelope-gas-pressure is applied. A comparison of the ideal gas EOS to the numerical Saumon-Chabrier EOS shows disagreement for the $\log T=$ 2.1 isotherm and densities above $\varrho=40 \mathrm{~kg} \mathrm{~m}^{-3}$. This would indicate that the non-ideal EOS is needed for high-density effective cores.
It has been noted that manifold properties are insensitive to variation of orbital distance $a$ or mass of the parent star $M_{\star}$, as long as $a M_{\star}^{-3}=$ const holds (cf. Sect. 3.8.2). Also, solutions whose envelope temperature and core mass obey relation (17) are found to be the same, if appropriate units for mass (i.e. core mass) and length (i.e. core radius) are used. This indicates the existence of analytic solutions for some envelope regimes, through certain dimensionless scaling variables. Such a treatment is, however, out of the scope of the present paper.

An envelope gas temperature is equal to the nebula $T$ throughout the protoplanet, and that certainly underestimates the thermal pressure and hence reduces the values for the critical core mass. However, from Eq. (17), one can show that for a more realistic estimate of the envelope temperature representative for the young planets $(5000 \mathrm{~K})$ critical core mass values are overestimated $\left(\sim 24.5 M_{\oplus}\right)$, because of envelope isothermality/lack of an energy transport equation and use of ideal-gas EOS, when compared to canonical critical core mass values from protoplanetary models with detailed microphysics.

Both the local and the global critical core masses signal the end of the availability of the hydrostatic solutions. In the case of the local critical core mass, non-availability holds for a small region of the parameter space around the local critical core mass, while for the global critical core mass this is true for every core larger than the critical core mass. The significant difference between the two types of critical core mass is that, at the global critical core mass (and above), the non-isothermal effects are crucial in shaping the structure of the protoplanetary envelopes, and are present throughout the parameter space. These non-isothermal effects are important for determining the details of the dynamical disk-planet interaction.

The critical core mass values obtained in this model are almost two orders of magnitude smaller than the canonical critical core masses which incorporate detailed energy transfer. Thus, if subcritical or just-critical regimes of a dynamical diskplanet interaction are to be investigated through a model that is locally isothermal, the planet mass should be set appropriately. Most of the present locally-isothermal disk-planet models (e.g. Kley 1999; D'Angelo et al. 2002, 2003; Nelson \& Papaloizou 2004) operate with planets which should be deep in the supercritical regime.

A solution set from our model encompasses solutions that are reminiscent of the planets in the various stages of evolution (from small rocks embedded in the dilute nebula to the mature planets as we know them), and of various configurations (the telluric planets of region I in Fig. 2, and the gas giants of region II). The "nebula" and "mature planet" regimes are the physically intuitive beginning and end phase of planetary evolution. However, the "protoplanet" regime presents us with an interesting region in parameter space, where planets could make the transition from "infancy" to "maturity". Depending on the detailed structure and the dynamics of the surrounding nebula, it is easy to conceive a standard scenario of planet formation. That is, the accretion of nebula gas onto a supercritical protoplanet. Other scenarios could be imagined as well, e.g. a massive protoplanet could release a major part of its envelope to reach the appropriate equilibrium, or it could dramatically condense its otherwise mostly gaseous envelope. Amounts of 
dust in the environment will doubtless play a very important role in the process.

In conclusion, several important features of the solution set have to be mentioned:

1. Two basic types of envelope equilibria are found for protoplanets:

- uniform; the density of the envelope gas drops weakly from the core to the outer boundary;

- compact; a dense gas layer forming an effective core, and a very low, exponentially decreasing, gas density further out.

Both types can be self-gravitating or non-self-gravitating, dividing the solution manifold into four distinct regions.

2. As a consequence of the envelope's self-gravitating effect, a wide range of possible envelope solutions exists.

3. We have developed a new concept for the global static critical core mass, which marks the contact point of all four qualitatively different types of protoplanets. This concept is based on a qualitative change of the envelope properties while considering a complete set of available solutions (a solution manifold), as opposed to the critical core mass definitions which are valid only for a solution subset fitting a particular nebula.

4. For every subcritical core there are at least two envelope solutions possible (a self-gravitating one and a non-selfgravitating one) for a given nebula, and for a certain nebula parameters the number of the possible envelope solutions can be even larger. Such nebulae also have multiple (local) critical core masses.

5. The global static critical core mass value is shown to decrease with increasing orbital distance $a$, mainly because of the decrease in the temperature of the surrounding nebula.

\section{References}

Bodenheimer, P., \& Pollack, J. B. 1986, Icarus, 67, 391

Bodenheimer, P., Hubickyj, O., \& Lissauer, J. J. 2000, Icarus, 143, 2

Carnahan, N. F., \& Starling, K. E. 1969, J. Chem. Phys., 51, 635

D’Angelo, G., Henning, T., \& Kley, W. 2002, A\&A, 385, 647

D'Angelo, G., Kley, W., \& T., Henning 2003, ApJ, 586, 540

Garvan, F. 2001, The Maple Book (Chapman \& Hall)

Guillot, T. 1999, Science, 286, 72

Hayashi, C., Nakazawa, K., \& Nakagawa, Y. 1985, Protostars and Planets II, 1100

Ikoma, M., Emori, H., \& Nakazawa, K. 2001, ApJ, 553, 999

Kippenhahn, R., \& Weigert, A. 1991, Stellar Structure and Evolution (Springer-Verlag)

Kley, W. 1999, MNRAS, 303, 696

Kusaka, T., Nakano, T., \& Hayashi, C. 1970, Prog. Theor. Phys., 44, 1580

Mizuno, H., Nakazawa, K., \& Hayashi, C. 1978, Prog. Theor. Phys., 60,699

Mizuno, H. 1980, Prog. Theor. Phys., 64, 544

Nelson, R. P., \& Papaloizou, J. C. B. 2004, MNRAS, 350, 849

Perri, F., \& Cameron, A. G. W. 1974, Icarus, 22, 416

Sasaki, S. 1989, A\&A, 215, 177

Shu, F. H. 1992, The Physics of Astrophysics, Vol. II (University Science Books)

Saumon, D., Chabrier, G., \& van Horn, H. M. 1995, ApJS, 99, 713

Stahl, B., Kiessling, M. K.-H., \& Schindler, K. 1995, Planet. Space Sci., 43, 271

Stevenson, D. J. 1982, Planet. Space Sci., 30, 755

Schwarzschild, M. 1957, Structure and Evolution of the Stars (Princeton University Press)

Wuchterl, G. 1991a, Icarus, 91, 39

Wuchterl, G. 1991b, Icarus, 91, 53

Wuchterl, G. 1993, Icarus, 106, 323

Wuchterl, G., Guillot, T., \& Lissauer, J. J. 2000, Protostars and Planets IV, 1081 